PROCEEDINGS OF THE

AMERICAN MATHEMATICAL SOCIETY

Volume 134, Number 11, November 2006, Pages 3143-3151

S 0002-9939(06)08369-9

Article electronically published on May 18, 2006

\title{
SIMULTANEOUS NON-VANISHING OF TWISTS
}

\author{
AMIR AKBARY
}

(Communicated by Wen-Ching Winnie Li)

\begin{abstract}
Let $f$ be a newform of even weight $k$, level $M$ and character $\psi$ and let $g$ be a newform of even weight $l$, level $N$ and character $\eta$. We give a generalization of a theorem of Elliott, regarding the average values of Dirichlet $L$-functions, in the context of twisted modular $L$-functions associated to $f$ and $g$. Using this result, we find a lower bound in terms of $Q$ for the number of primitive Dirichlet characters modulo prime $q \leq Q$ whose twisted product $L$-functions $L_{f, \chi}\left(s_{0}\right) L_{g, \chi}\left(s_{0}\right)$ are non-vanishing at a fixed point $s_{0}=\sigma_{0}+i t_{0}$ with $\frac{1}{2}<\sigma_{0} \leq 1$.
\end{abstract}

\section{INTRODUCTION}

Let $L_{\chi}(s)=\sum_{n=1}^{\infty} \chi(n) n^{-s}$ be the Dirichlet $L$-function associated to a Dirichlet character $\chi$. In [E], Elliott proved the following.

Theorem. Let $Q \geq 2$ be a real number, and $s_{0}=\sigma_{0}+i t_{0}$ a complex number in the half-plane $\sigma_{0}>\frac{1}{2}$. Then we have

$$
\sum_{p \leq Q} \sum_{\chi \neq \chi_{0}}\left|L_{\chi}\left(s_{0}\right)\right|^{2}=\frac{Q^{2}}{2 \log Q} \zeta\left(2 \sigma_{0}\right)+O\left(\frac{Q^{2}}{(\log Q)^{2}}\right)
$$

as $Q \rightarrow \infty$. Here the inner sum is taken over all non-principal characters $(\bmod p)$, for each prime $p$, and the outer sum over all prime numbers not exceeding $Q$.

Our first goal in this paper is to give a generalization of this theorem in the context of twisted modular $L$-functions. Let $S_{k}\left(\Gamma_{0}(M), \psi\right)$ be the space of holomorphic cusp forms of even weight $k$, level $M$ and character $\psi$. For $f \in S_{k}\left(\Gamma_{0}(M), \psi\right)$, let

$$
f(z)=\sum_{n=1}^{\infty} a_{f}(n) n^{\frac{k-1}{2}} e^{2 \pi i n z}
$$

be the Fourier expansion of $f$ at $i \infty$. Let $\chi$ be a primitive Dirichlet character mod $q$ with $(q, M)=1$. Then the twisted $L$-function associated to $f$ and $\chi$ is defined (for $\operatorname{Re}(s)>1$ ) by

$$
L_{f, \chi}(s)=\sum_{n=1}^{\infty} \frac{a_{f}(n) \chi(n)}{n^{s}}
$$

Received by the editors August 16, 2004 and, in revised form, June 9, 2005.

2000 Mathematics Subject Classification. Primary 11F67.

This research was partially supported by NSERC.

(C)2006 American Mathematical Society Reverts to public domain 28 years from publication 
Let

and

$$
L_{\infty, k}(s)=(2 \pi)^{-s} \Gamma\left(\frac{k-1}{2}+s\right)
$$

$$
\Lambda_{f, \chi}(s)=(q \sqrt{M})^{s} L_{\infty, k}(s) L_{f, \chi}(s) .
$$

Then it is known that $\Lambda_{f, \chi}(s)$ is entire, and if $f$ is a newform (in Atkin-Lehner sense), it satisfies the functional equation

$$
\Lambda_{f, \chi}(s)=\epsilon_{f, \chi} \Lambda_{\bar{f}, \bar{\chi}}(1-s),
$$

where $\bar{f}$ is the conjugate newform in $S_{k}\left(\Gamma_{0}(M), \bar{\psi}\right)$. Here

$$
\epsilon_{f, \chi}=\epsilon_{f} \psi(q) \chi(M) \tau(\chi)^{2} q^{-1},
$$

where $\left|\epsilon_{f}\right|=1$ and $\tau(\chi)$ is the Gauss sum. Note that $\left|\epsilon_{f, \chi}\right|=1$.

We recall that for $f \in S_{k}\left(\Gamma_{0}(M), \psi\right)$ and $g \in S_{l}\left(\Gamma_{0}(N), \eta\right)$ the Rankin-Selberg convolution $L$-function is defined (for $\operatorname{Re}(s)>1$ ) by

$$
L(f \otimes g, s)=\sum_{n=1}^{\infty} \frac{a_{f}(n) \bar{b}_{g}(n)}{n^{s}} .
$$

The following can be considered as a modular analogue of the above theorem of Elliott.

Theorem 1.1. Let $f \in S_{k}\left(\Gamma_{0}(M), \psi\right)$ and $g \in S_{l}\left(\Gamma_{0}(N), \eta\right)$ be newforms. Let $Q \geq 2$ and let $s_{0}=\sigma_{0}+i t_{0}$ be a complex number with $\sigma_{0}>\frac{1}{2}$. Then we have

$$
\sum_{\substack{q \leq Q, q \\(q, M N)=1}} \sum_{\substack{p r i m e \\(\bmod }}^{\star} L_{f, \chi}\left(s_{0}\right) \overline{L_{g, \chi}\left(s_{0}\right)}=\frac{Q^{2}}{2 \log Q} \frac{\phi(M N)}{M N} L\left(f \otimes g, 2 \sigma_{0}\right)+O\left(\frac{Q^{2}}{(\log Q)^{2}}\right)
$$

where the inner sum is taken over the primitive characters modulo prime $q$. The implied constant depends on $f, g$ and $s_{0}$. Here, $\phi$ is the Euler function.

In proving Theorem 1.1, we first find an asymptotic formula for the values $L_{f, \chi}\left(s_{0}\right) \overline{L_{g, \chi}\left(s_{0}\right)}$ on average when $\chi$ varies on the set of primitive characters modulo a fixed positive integer $q$ (see Proposition 2.5). This result generalizes a theorem of Stefanicki ([S], Theorem 2(a)).

Theorem 1.1 has an interesting application in the problem of non-vanishing of twisted $L$-functions inside the critical strip. In Proposition 3.1, by employing the large sieve inequality for characters, we establish an upper bound for the mean square of the values $\left|L_{f, \chi}\left(s_{0}\right) \overline{L_{g, \chi}\left(s_{0}\right)}\right|$. Together, Theorem 1.1 and Proposition 3.1 imply the following.

Theorem 1.2. Let $f \in S_{k}\left(\Gamma_{0}(M), \psi\right)$ and $g \in S_{l}\left(\Gamma_{0}(N), \eta\right)$ be newforms. Let $s_{0}=\sigma_{0}+i t_{0}$ be a fixed point in the strip $\frac{1}{2}<\sigma_{0} \leq 1$. Then we have

$$
\#\left\{\chi \mid \operatorname{conductor}(\chi) \text { a prime } \leq Q \text { and } L_{f, \chi}\left(s_{0}\right) L_{g, \chi}\left(s_{0}\right) \neq 0\right\} \gg \frac{Q^{2}}{(\log Q)^{4}}
$$

as $Q \rightarrow \infty$. The implied constant depends on $f, g$ and $s_{0}$.

This theorem should be compared to some non-vanishing results in the theory of automorphic forms. To explain the connection, let $F$ be a number field, let $S$ be a finite set of places of $F$, and let $\pi$ be a unitary cuspidal automorphic representation of GL $(n)$ over $F$. Let $s_{0}=\sigma_{0}+i t_{0}$ be a fixed point in the complex 
plane. Then Rohrlich $[\mathbb{R}$ proved that for $n=1$ and 2 there are infinitely many primitive ray class characters $\chi$ of $F$ such that $\chi$ is unramified at the places in $S$ and $L\left(\pi \otimes \chi, s_{0}\right) \neq 0$. For $n \geq 3$, Barthel and Ramakrishnan [BR] proved that the same result remains true as long as $\pi$ is tempered (i.e. satisfies the Ramanujan conjecture) and $\sigma_{0}>1-\frac{2}{n+1}$ (see also [LRS for a related result). For automorphic representations of $\mathrm{GL}(4)$ over $\mathbb{Q}$ (the case that is related to this paper) the result of Barthel and Ramakrishnan states that for $\sigma_{0}>\frac{3}{5}$ there are infinitely many primitive Dirichlet characters such that $L\left(\pi \otimes \chi, s_{0}\right) \neq 0$. Note that our nonvanishing result (Theorem 1.2) surpasses the bound $\frac{3}{5}$. This is due to the fact that we are dealing with the product of two twisted GL(2) $L$-functions $\left(L_{f, \chi}(s) \overline{L_{g, \chi}(s)}\right)$ and thus the Gauss sums associated to the functional equations of these two $L$ functions cancel each other (see Lemma 2.2). Therefore the contributions from the sums corresponding to $1-s_{0}$ in Lemma 2.2 can be dealt with in ways similar to the sums corresponding to $s_{0}$. This enables us to prove a non-vanishing result in the half plane $\sigma_{0}>\frac{1}{2}$. In fact a similar result should be true on the line $\sigma_{0}=\frac{1}{2}$, however establishing such a result needs a more elaborate treatment of the error terms in Proposition 2.5.

In the next two sections we prove the above theorems.

\section{Proof of Theorem 1.1}

Let $k \geq l$ and $s_{0}=\sigma_{0}+i t_{0}$. We set

$$
P_{\chi}\left(s_{0}\right)=L_{f, \chi}\left(s_{0}\right) \overline{L_{g, \chi}\left(s_{0}\right)} .
$$

We first derive an asymptotic formula for $\sum_{\chi} P_{\chi}\left(s_{0}\right)$ as $\chi$ varies over the primitive characters $\bmod q$. Here we do not assume that $q$ is a prime. Let

$$
Z_{s_{0}}(x)=\frac{1}{2 \pi i} \int_{(1)} L_{\infty, k}\left(s+s_{0}\right) L_{\infty, l}\left(s+\bar{s}_{0}\right) x^{-s} \frac{d s}{s} .
$$

Writing the integral representations of the $\Gamma$ functions in the expression for $Z_{s_{0}}(x)$ and interchanging the order of integration, we arrive at

$$
Z_{s_{0}}(x)=(2 \pi)^{-2 \sigma_{0}} \int_{0}^{\infty} t_{1}^{\frac{k-1}{2}+s_{0}-1} e^{-t_{1}}\left(\int_{\frac{4 \pi^{2} x}{t_{1}}}^{\infty} t_{2}^{\frac{l-1}{2}+\bar{s}_{0}-1} e^{-t_{2}} d t_{2}\right) d t_{1} .
$$

Note that this representation for $Z_{s_{0}}(x)$ shows that $\left|Z_{s_{0}}(x)\right| \leq Z_{\sigma_{0}}(x)$. Moreover by integration by parts we can find an expression for $Z_{s_{0}}(x)$ in terms of $K$-Bessel functions. Applying the standard bounds for $K$-Bessel functions yields

$$
\left|Z_{s_{0}}(x)\right| \ll\left\{\begin{array}{cc}
1, & x \leq 1, \\
x^{\frac{k}{4}+\frac{l}{4}+\sigma_{0}-\frac{5}{4}} e^{-4 \pi \sqrt{x}}, & x>1
\end{array}\right.
$$

(see [A], Lemmas 6.2 and 6.3, for details).

We next represent $P_{\chi}\left(s_{0}\right)$ as a sum of two rapidly convergent series.

Lemma 2.1. Let $f \in S_{k}\left(\Gamma_{0}(M), \psi\right)$ and $g \in S_{l}\left(\Gamma_{0}(N), \eta\right)$ be newforms. Suppose that $\chi$ is a primitive Dirichlet character modulo $q$ with $(q, M N)=1$. Then

$$
L_{\infty, k}\left(s_{0}\right) L_{\infty, l}\left(\bar{s}_{0}\right) P_{\chi}\left(s_{0}\right)=S_{f, g}\left(s_{0}\right)+\epsilon_{f, \chi} \epsilon_{\bar{g}, \bar{\chi}}\left(q^{2} \sqrt{M N}\right)^{1-2 \sigma_{0}}\left(\frac{N}{M}\right)^{i t_{0}} \hat{S}_{f, g}\left(1-s_{0}\right),
$$


where

$$
S_{f, g}\left(s_{0}\right)=\sum_{m, n \geq 1} \frac{a_{f}(m) \bar{a}_{g}(n)}{(m n)^{\sigma_{0}}}\left(\frac{n}{m}\right)^{i t_{0}} Z_{s_{0}}\left(\frac{m n}{q^{2} \sqrt{M N}}\right) \chi(m) \bar{\chi}(n)
$$

and

$$
\hat{S}_{f, g}\left(1-s_{0}\right)=\sum_{m, n \geq 1} \frac{\bar{a}_{f}(m) a_{g}(n)}{(m n)^{1-\sigma_{0}}}\left(\frac{m}{n}\right)^{i t_{0}} Z_{1-s_{0}}\left(\frac{m n}{q^{2} \sqrt{M N}}\right) \bar{\chi}(m) \chi(n) .
$$

Proof. We have

$S_{f, g}\left(s_{0}\right)=\frac{1}{2 \pi i} \int_{(1)} L_{\infty, k}\left(s+s_{0}\right) L_{f, \chi}\left(s+s_{0}\right) L_{\infty, l}\left(s+\bar{s}_{0}\right) L_{\bar{g}, \bar{\chi}}\left(s+\bar{s}_{0}\right)\left(q^{2} \sqrt{M N}\right) \frac{d s}{s}$.

Moving the line of integration to the left of zero and calculating the residue at $s=0$, along with application of (11) result in

$$
\begin{aligned}
& S_{f, g}\left(s_{0}\right)=L_{\infty, k}\left(s_{0}\right) L_{\infty, l}\left(\bar{s}_{0}\right) P_{\chi}\left(s_{0}\right)+\epsilon_{f, \chi} \epsilon_{\bar{g}, \bar{\chi}}\left(q^{2} \sqrt{M N}\right)^{1-2 \sigma_{0}}\left(\frac{N}{M}\right)^{i t_{0}} \\
& \times \frac{1}{2 \pi i} \int_{(-1)} L_{\infty, k}\left(1-s-s_{0}\right) L_{\bar{f}, \bar{\chi}}\left(1-s-s_{0}\right) \\
& L_{\infty, l}\left(1-s-\bar{s}_{0}\right) L_{g, \chi}\left(1-s-\bar{s}_{0}\right)\left(q^{2} \sqrt{M N}\right)^{-s} \frac{d s}{s} .
\end{aligned}
$$

Now changing $s$ to $-s$ yields the result.

From now on for simplicity we let $L_{\infty}\left(s_{0}\right)=L_{\infty, k}\left(s_{0}\right) L_{\infty, l}\left(\bar{s}_{0}\right)$. Next we average $P_{\chi}\left(s_{0}\right)$ over all primitive Dirichlet characters modulo $q$. We have

Lemma 2.2. Let $q \not \equiv 2(\bmod 4)$ and $(q, M N)=1$. Then

$$
\begin{aligned}
L_{\infty}\left(s_{0}\right) & \sum_{\chi(\bmod q)}^{\star} P_{\chi}\left(s_{0}\right) \\
\quad= & \sum_{d \mid q} \mu\left(\frac{q}{d}\right) \phi(d)\left(S_{f, g}^{d}\left(s_{0}\right)+\epsilon_{f} \epsilon_{\bar{g}} \psi \bar{\eta}(q)\left(q^{2} \sqrt{M N}\right)^{1-2 \sigma_{0}}\left(\frac{N}{M}\right)^{i t_{0}} \hat{S}_{f, g}^{d}\left(1-s_{0}\right)\right),
\end{aligned}
$$

where $\mu$ is the Möbius function, $\phi$ is the Euler function,

$$
S_{f, g}^{d}\left(s_{0}\right)=\sum_{\substack{m, n,(m n, q)=1 \\ m \equiv n(\bmod d)}} \frac{a_{f}(m) \bar{a}_{g}(n)}{(m n)^{\sigma_{0}}}\left(\frac{n}{m}\right)^{i t_{0}} Z_{s_{0}}\left(\frac{m n}{q^{2} \sqrt{M N}}\right),
$$

and

$$
\hat{S}_{f, g}^{d}\left(1-s_{0}\right)=\sum_{\substack{m, n,(m n, q)=1 \\ N m \equiv M n(\bmod d)}} \frac{\bar{a}_{f}(m) a_{g}(n)}{(m n)^{1-\sigma_{0}}}\left(\frac{m}{n}\right)^{i t_{0}} Z_{1-s_{0}}\left(\frac{m n}{q^{2} \sqrt{M N}}\right) .
$$

Proof. From Lemma 2.1 we have

$$
\begin{aligned}
& L_{\infty}\left(s_{0}\right) \sum_{\chi(\bmod q)}^{\star} P_{\chi}\left(s_{0}\right) \\
& =\sum_{\chi(\bmod q)}^{\star}\left(S_{f, g}\left(s_{0}\right)+\epsilon_{f} \epsilon_{\bar{g}} \psi \bar{\eta}(q) \chi(M) \bar{\chi}(N)\left(q^{2} \sqrt{M N}\right)^{1-2 \sigma_{0}}\left(\frac{N}{M}\right)^{i t_{0}} \hat{S}_{f, g}\left(1-s_{0}\right)\right) .
\end{aligned}
$$


Note that $\epsilon_{f, \chi} \epsilon_{\bar{g}, \bar{\chi}}=\epsilon_{f} \epsilon_{\bar{g}} \psi \bar{\eta}(q) \chi(M) \bar{\chi}(N)$. To simplify the above expression, we need to evaluate $\sum_{\chi(\bmod q)}^{\star} \chi(m) \bar{\chi}(n)$ and $\sum_{\chi(\bmod q)}^{\star} \chi(M n) \bar{\chi}(N m)$. Let

$$
h_{m, n}(q)=\sum_{\chi(\bmod q)}^{\star} \chi(m) \bar{\chi}(n) .
$$

We have

$$
\sum_{d \mid q} h_{m, n}(d)=\sum_{\chi(\bmod q)} \chi(m) \bar{\chi}(n)=\left\{\begin{array}{cc}
\phi(q) & \text { if } m \equiv n(\bmod q) \\
0 & \text { otherwise }
\end{array}\right.
$$

Now applying the Möbius inversion formula ( $\underline{M}$, Section 1.1) on the above identity yields

$$
\sum_{\chi(\bmod q)}^{\star} \chi(m) \bar{\chi}(n)=h_{m, n}(q)=\sum_{d \mid(q, m-n)} \mu\left(\frac{q}{d}\right) \phi(d) .
$$

Applying this and a similar identity for $\sum_{\chi(\bmod q)}^{\star} \chi(M n) \bar{\chi}(N m)$ in the expression for $L_{\infty}\left(s_{0}\right) \sum_{\chi(\bmod q)}^{\star} P_{\chi}\left(s_{0}\right)$ at the beginning of the proof imply the result.

Next we find an asymptotic for the terms in $S_{f, g}^{d}\left(s_{0}\right)$ corresponding to $m=n$. To explain our result we need to introduce a notation. We know that for any prime $p$, $a_{f}(p)=\alpha_{f, 1}(p)+\alpha_{f, 2}(p)$ and $a_{g}(p)=\alpha_{g, 1}(p)+\alpha_{g, 2}(p)$, where $\alpha_{f, 1}(p) \alpha_{f, 2}(p)=\psi(p)$ and $\alpha_{g, 1}(p) \alpha_{g, 2}(p)=\eta(p)$. Let

$$
R_{q}(s)=\prod_{p \mid q}\left(1-\frac{\psi \bar{\eta}(p)}{p^{2 s}}\right)^{-1} \prod_{i=1}^{2} \prod_{j=1}^{2}\left(1-\frac{\alpha_{f, i}(p) \bar{\alpha}_{g, j}(p)}{p^{s}}\right) .
$$

Lemma 2.3. Let $f, g$ and $s_{0}$ be as Theorem 1.1. Then

$$
\sum_{n,(n, q)=1} \frac{a_{f}(n) \bar{a}_{g}(n)}{n^{2 \sigma_{0}}} Z_{s_{0}}\left(\frac{n^{2}}{q^{2} \sqrt{M N}}\right) \sim L_{\infty}\left(s_{0}\right) L\left(f \otimes g, 2 \sigma_{0}\right) R_{q}\left(2 \sigma_{0}\right)
$$

as $q \rightarrow \infty$.

Proof. From the definition of $Z_{s_{0}}(x)$, we have

$$
\begin{gathered}
\sum_{n,(n, q)=1} \frac{a_{f}(n) \bar{a}_{g}(n)}{n^{2 \sigma_{0}}} Z_{s_{0}}\left(\frac{n^{2}}{q^{2} \sqrt{M N}}\right) \\
=\frac{1}{2 \pi i} \int_{(1)} L_{\infty, k}\left(s+s_{0}\right) L_{\infty, l}\left(s+\bar{s}_{0}\right) L\left(f \otimes g, 2 s+2 \sigma_{0}\right) R_{q}\left(2 s+2 \sigma_{0}\right)\left(q^{2} \sqrt{M N}\right) \frac{d s}{s} .
\end{gathered}
$$

Moving the line of integration to the left of zero implies the result.

The next lemma gives an estimation for the off-diagonal terms in $S_{f, g}^{d}\left(s_{0}\right)$.

Lemma 2.4. Let $\epsilon>0$ be arbitrary. Then

$$
\sum_{d \mid q} \mu\left(\frac{q}{d}\right) \phi(d) \sum_{\substack{m, n,(m n, q)=1 \\ m \equiv n(\bmod d), m \neq n}} \frac{a_{f}(m) \bar{a}_{g}(n)}{(m n)^{\sigma_{0}}}\left(\frac{n}{m}\right)^{i t_{0}} Z_{s_{0}}\left(\frac{m n}{q^{2} \sqrt{M N}}\right)=O\left(q^{2-2 \sigma_{0}+\epsilon}\right) .
$$

The implied constant depends on $f, g, s_{0}$ and $\epsilon$. 
Proof. We closely follow Section 3.2 of $[\underline{\mathrm{S}}$. First of all we recall Rankin-Shiu's estimate for the sum of Fourier coefficients of modular forms. Let $d \neq 1$ and $(n, d)=1$. Then for a newform $g$ we have

$$
\sum_{\substack{n \leq x \\ n \equiv m(\bmod d)}}\left|a_{g}(n)\right| \ll \frac{x}{\phi(d)}(\log x)^{-\epsilon_{1}}
$$

as $x \rightarrow \infty$, where $x>d^{\alpha}$ for $1<\alpha<2$. Here, $0<\epsilon_{1} \leq \delta \simeq 0.06 \ldots$ is arbitrary, and the bound is uniform in $m, d$ and $\alpha$ (see [S], page 5 for details). We use this together with Rankin's estimate [RA1]

$$
\sum_{m \leq x}\left|a_{f}(m)\right| \ll x(\log x)^{-\delta}
$$

to bound the inner sum in the statement of the lemma. Let $1<\alpha<\frac{10}{9}$ be a fixed number. We only need to find estimates for the following ranges of $m$ and $n$ :

(i) $n>d^{\alpha}$.

(ii) $d^{\frac{4}{5} \alpha} \leq n<m \leq d^{\alpha}$.

(iii) $n<d^{\frac{4}{5} \alpha}$ and $d \leq m \leq d^{\alpha}$.

Now we estimate the inner sum in the statement of the lemma in each case.

(i) We assume $n>d^{\alpha}$. By employing Rankin-Shiu's and Rankin's estimates, bounds for $Z_{\sigma_{0}}(x)$ and partial summation we have

$$
\sum_{\substack{m \geq \frac{q^{2} \sqrt{M N}}{d d^{d}} \\(m, q)=1}} \frac{\left|a_{f}(m)\right|}{m^{\sigma_{0}}} \sum_{\substack{n>d^{\alpha},(n, q)=1 \\ n \equiv m(\bmod d)}} \frac{\left|a_{g}(n)\right|}{n^{\sigma_{0}}} Z_{\sigma_{0}}\left(\frac{m n}{q^{2} \sqrt{M N}}\right) \ll \frac{1}{\phi(d)}\left(q^{2} \sqrt{M N}\right)^{1-\sigma_{0}}
$$

and

$$
\begin{gathered}
\sum_{\substack{m<q^{2} \sqrt{M N} \\
(m, q)=1}} \frac{\left|a_{f}(m)\right|}{m^{\sigma_{0}}} \sum_{\substack{n>d^{\alpha},(n, q)=1 \\
n \equiv m(\bmod d)}} \frac{\left|a_{g}(n)\right|}{n^{\sigma_{0}}} Z_{\sigma_{0}}\left(\frac{m n}{q^{2} \sqrt{M N}}\right) \\
\ll \frac{1}{\phi(d)}\left(q^{2} \sqrt{M N}\right)^{1-\sigma_{0}}\left(\log \left(d^{-\alpha} q^{2} \sqrt{M N}\right)\right)^{1-\delta} .
\end{gathered}
$$

(ii) Next we consider the range $d^{\frac{4}{5} \alpha} \leq n<m \leq d^{\alpha}$. We recall from the RankinSelberg theory [RA2] the asymptotic formula

$$
\sum_{n \leq x}\left|a_{g}(n)\right|^{2}=c_{g} x+O\left(x^{\frac{3}{5}}\right),
$$

where $c_{g}$ is a constant depending only on $g$. By employing the Cauchy-Schwarz inequality and the above asymptotic we have

$$
\sum_{n \leq x}\left|a_{f}(n+d t) a_{g}(n)\right| \ll x
$$


uniformly for $x \gg(d t)^{\frac{3}{5}}$. Now by writing $m=n+d t$ for $1 \leq t \leq d^{\alpha-1}$, and applying partial summation, we get the following estimation of the inner sum:

$$
\begin{aligned}
& \sum_{1 \leq t \leq d^{\alpha-1}} \sum_{d^{\frac{4}{5} \alpha}<n \leq \frac{q^{2} \sqrt{M N}}{d t}} \frac{\left|a_{f}(n+d t) a_{g}(n)\right|}{(n+d t)^{\sigma_{0}} n^{\sigma_{0}}} Z_{\sigma_{0}}\left(\frac{(n+d t) n}{q^{2} \sqrt{M N}}\right) \\
& \ll \sum_{1 \leq t \leq d^{\alpha-1}} \frac{\left(q^{2} \sqrt{M N}\right)^{1-\sigma_{0}}}{d t} \ll \frac{1}{d}\left(q^{2} \sqrt{M N}\right)^{1-\sigma_{0}} \log d .
\end{aligned}
$$

A similar estimation is true for the range $\frac{q^{2} \sqrt{M N}}{d t}<n \leq d^{\alpha}$.

(iii) Finally we consider $m$ and $n$ 's in the range $n<d^{\frac{4}{5} \alpha}$ and $d \leq m \leq d^{\alpha}$. Note that since $1<\alpha<\frac{10}{9}$ and $\sigma_{0}>\frac{1}{2}$, we have $\frac{1}{2}-\frac{9}{10} \alpha\left(1-\sigma_{0}\right)>0$. We choose an $\epsilon$ such that $0<\epsilon<\frac{1}{2}-\frac{9}{10} \alpha\left(1-\sigma_{0}\right)$. By Deligne's bound for the Fourier coefficients of newforms we have

$$
\left|a_{f}(m) a_{g}(n)\right| \ll_{\epsilon} d^{\epsilon} .
$$

By applying this bound we have

$$
\sum_{\substack{n<d^{\frac{4}{5} \alpha}, d \leq m \leq d \\ m \equiv n(\bmod d)}} \frac{\left|a_{f}(m) a_{g}(n)\right|}{(m n)^{\sigma_{0}}} Z_{\sigma_{0}}\left(\frac{m n}{q^{2} \sqrt{M N}}\right) \ll d^{\frac{9}{5} \alpha\left(1-\sigma_{0}\right)-1+\epsilon} \ll d^{-\epsilon} .
$$

Applying estimates of (i), (ii) and (iii) to the inner sum in the statement of the lemma implies the result.

Combining the results of Lemmas 2.2, 2.3 and 2.4 we have the following.

Proposition 2.5. Let $f, g$ and $s_{0}$ be as Theorem 1.1 and let $\epsilon>0$ be arbitrary. Let $q \not \equiv 2(\bmod 4)$ and $(q, M N)=1$. We have

$$
\sum_{\chi(\bmod q)}^{\star} P_{\chi}\left(s_{0}\right)=L\left(f \otimes g, 2 \sigma_{0}\right) R_{q}\left(2 \sigma_{0}\right) \sum_{d \mid q} \mu\left(\frac{q}{d}\right) \phi(d)+O\left(q^{2-2 \sigma_{0}+\epsilon}\right) .
$$

The implied constant depends on $f, g, s_{0}$ and $\epsilon$.

Proof. First of all note that a result similar to Lemma 2.4 is also true for the offdiagonal terms in $\hat{S}_{f, g}^{d}\left(1-s_{0}\right)$, and in this case the corresponding sum is bounded by $q^{2 \sigma_{0}+\epsilon}$. For the diagonal terms in $\hat{S}_{f, g}^{d}\left(1-s_{0}\right)$ (ones corresponding to $N m=M n$ ), by applying Deligne's bound for Fourier coefficients of new forms we have

$$
\begin{aligned}
& \sum_{\substack{m, n,(m n, q)=1 \\
N m=M n}} \frac{\left|a_{f}(m) a_{g}(n)\right|}{(m n)^{1-\sigma_{0}}} Z_{1-\sigma_{0}}\left(\frac{m n}{q^{2} \sqrt{M N}}\right) \\
& \quad \ll_{M, N} \sum_{n,(n, q)=1} \frac{n^{\epsilon}}{n^{2\left(1-\sigma_{0}\right)}} Z_{1-\sigma_{0}}\left(\frac{M n^{2}}{q^{2} N \sqrt{M N}}\right) . \ll q^{2 \sigma_{0}-1+\epsilon} .
\end{aligned}
$$

Applying these estimates together with Lemmas 2.3 and 2.4 in Lemma 2.2 will imply the desired result.

Proof of Theorem 1.1. We sum the asymptotic formula given in Lemma 2.5 over primes $q \leq Q$ where $(q, M N)=1$. Note that for $q$ prime, $R_{q}\left(2 \sigma_{0}\right)=1+O\left(q^{-2 \sigma_{0}}\right)$ and $\sum_{d \mid q} \mu\left(\frac{q}{d}\right) \phi(d)=q-2$. Now the result follows from the prime number theorem. 


\section{Proof of Theorem 1.2}

First of all in Lemma 2.1 let

$$
b_{u}=\sum_{u=m n} a_{f}(m) \bar{a}_{g}(n) \chi(m) \bar{\chi}(n)\left(\frac{n}{m}\right)^{i t_{0}} .
$$

Note that by Deligne's bound for Fourier coefficients of newforms we have

$$
\left|b_{u}\right| \ll_{\epsilon} u^{\epsilon} .
$$

So by Lemma 2.1 for given $X$ we have

$$
\begin{aligned}
L_{\infty}\left(s_{0}\right) P_{\chi}\left(s_{0}\right) & =\sum_{u \leq X} \frac{b_{u}}{u^{\sigma_{0}}} Z_{s_{0}}\left(\frac{u}{q^{2} \sqrt{M N}}\right)+\sum_{u \geq X} \frac{b_{u}}{u^{\sigma_{0}}} Z_{s_{0}}\left(\frac{u}{q^{2} \sqrt{M N}}\right) \\
& +\epsilon_{f, \chi} \epsilon_{\bar{g}, \bar{\chi}}\left(q^{2} \sqrt{M N}\right)^{1-2 \sigma_{0}}\left(\frac{N}{M}\right)^{i t_{0}} \sum_{u \leq X} \frac{\bar{b}_{u}}{u^{1-\sigma_{0}}} Z_{1-s_{0}}\left(\frac{u}{q^{2} \sqrt{M N}}\right) \\
& +\epsilon_{f, \chi} \epsilon_{\bar{g}, \bar{\chi}}\left(q^{2} \sqrt{M N}\right)^{1-2 \sigma_{0}}\left(\frac{N}{M}\right)^{i t_{0}} \sum_{u \geq X} \frac{\bar{b}_{u}}{u^{1-\sigma_{0}}} Z_{1-s_{0}}\left(\frac{u}{q^{2} \sqrt{M N}}\right) \\
& =L_{1}\left(s_{0}\right)+L_{2}\left(s_{0}\right)+L_{3}\left(s_{0}\right)+L_{4}\left(s_{0}\right) .
\end{aligned}
$$

From here we have

$$
\left|L_{\infty}\left(s_{0}\right)\right|^{2} \sum_{\substack{q \leq Q \\(q, M N)=1}} \frac{q}{\phi(q)} \sum_{\chi(\bmod q)}^{\star}\left|P_{\chi}\left(s_{0}\right)\right|^{2} \ll\left|L_{\infty}\left(s_{0}\right)\right|^{2} \sum_{i=1}^{4} \sum_{q \leq Q} \frac{q}{\phi(q)} \sum_{\chi(\bmod q)}^{\star}\left|L_{i}\left(s_{0}\right)\right|^{2} .
$$

Now let $X=\sqrt{M N} Q^{2}(\log Q)^{2}$. Then by employing bound (2) for $Z_{s_{0}}(x)$, we have

$$
\sum_{q \leq Q} \frac{q}{\phi(q)} \sum_{\chi(\bmod q)}^{\star}\left|L_{i}\left(s_{0}\right)\right|^{2} \ll Q^{-19}
$$

for $i=2,4$. We know that by the large sieve inequality for characters we have

$$
\sum_{q \leq Q} \frac{q}{\phi(q)} \sum_{\chi(\bmod q)}^{\star}\left|\sum_{u=1}^{X} a_{u} \chi(u)\right|^{2} \leq\left(X+3 Q^{2}\right) \sum_{u=1}^{X}\left|a_{u}\right|^{2}
$$

(see [D], page 160, Theorem 4). Let

$$
a_{u}=u^{-\sigma_{0}} Z_{s_{0}}\left(\frac{u}{q^{2} \sqrt{M N}}\right) \sum_{\substack{u=m n \\(n, q)=1}} a_{f}(m) \bar{a}_{g}(n) \bar{\chi}\left(n^{2}\right)\left(\frac{n}{m}\right)^{i t_{0}} .
$$

By Deligne's bound and (2) we have

$$
\left|a_{u}\right| \ll_{\epsilon} u^{-\sigma_{0}+\epsilon} \text {. }
$$

This bound together with the large sieve inequality imply that for $i=1$

$$
\sum_{q \leq Q} \frac{q}{\phi(q)} \sum_{\chi(\bmod q)}^{\star}\left|L_{i}\left(s_{0}\right)\right|^{2}=\sum_{q \leq Q} \frac{q}{\phi(q)} \sum_{\chi(\bmod q)}^{\star}\left|\sum_{u=1}^{X} a_{u} \chi(u)\right|^{2} \ll Q^{2}(\log Q)^{2} .
$$

The same bound is valid for $i=3$. Now applying (44) and (5) in (3) implies the following. 
Proposition 3.1. Let $f, g$ and $s_{0}$ be as Theorem 1.1. We have

$$
\sum_{\substack{q \leq Q \\(q, M N)=1}} \frac{q}{\phi(q)} \sum_{\chi(\bmod q)}^{\star}\left|P_{\chi}\left(s_{0}\right)\right|^{2} \ll Q^{2}(\log Q)^{2} .
$$

The implied constant depends on $f, g$ and $s_{0}$.

Proof of Theorem 1.2. By Cauchy-Schwarz inequality we have

$$
\#\left\{\chi \mid \text { conductor }(\chi) \text { a prime } \leq Q \text { and } P_{\chi}\left(s_{0}\right) \neq 0\right\} \geq \frac{\left|\sum_{\substack{q \leq Q, q \text { prime } \\(q, M N)=1}} \sum_{\chi(\bmod q)}^{\star} P_{\chi}\left(s_{0}\right)\right|^{2}}{\sum_{\substack{q \leq Q \\(q, M N)=1}} \frac{q}{\phi(q)} \sum_{\chi(\bmod q)}^{\star}\left|P_{\chi}\left(s_{0}\right)\right|^{2}} .
$$

The result follows from this inequality, Theorem 1.1 and Proposition 3.1.

\section{ACKNOWLEDGMENT}

The author would like to thank the referee for helpful comments and suggestions. I would also like to thank Hershy Kisilevsky for several helpful discussions related to this work.

\section{REFERENCES}

[A] A. Akbary, Non-vanishing of weight $k$ modular $L$-functions with large level, J. Ramanujan Math. Soc. 14 (1999), 37-54. MR.1700874(2000e:11067)

[BR] L. Barthel and D. Ramakrishnan, A nonvanishing result for twists of $L$-functions of GL(n), Duke Math. J. 74 (1994), 681-700. MR1277950 (95d:11062)

[D] H. Davenport, Multiplicative Number Theory, third edition, Springer, 2000. MR 1790423 (2001f:11001)

[LRS] W. Luo, Z. Rudnick, and P. Sarnak, On the generalized Ramanujan conjecture for GL( $n)$. In Automorphic forms, automorphic representations, and arithmetic (Fort Worth, TX, 1996), 301-310, Proc. Sympos. Pure Math., 66, Part 2, AMS, 1999. MR1703764(2000e:11072)

[E] P. D. T. A. Elliott, On the distribution of the values of Dirichlet $L$-series in the half plane $\sigma>\frac{1}{2}$, Indag. Math. 33 (1971), 222-234. MR0291100(45:194)

[M] M. R. Murty, Problems in Analytic Number Theory, Springer, 2001. MR.1803093 (2001k:11002)

[R] D. Rohrlich, Nonvanishing of $L$-functions for GL(2), Invent. Math. 97 (1989), 381-403. MR:1001846 (90g:11062)

[RA1] R. A. Rankin, Sums of powers of cusp form coefficients, II, Math. Ann. 272 (1985), 593-600. MR0807293 (87d:11032)

[RA2] R. A. Rankin, Contributions to the theory of Ramanujan's function $\tau(n)$ and similar arithmetical functions. II. The order of the Fourier coefficients of integral modular forms, Proc. Cambridge Phil. Soc. 35 (1939), 357-372. MR0000411(1:69d)

[S] T. Stefanicki, Non-vanishing of $L$-functions attached to automorphic representations of GL(2) over Q, J. Reine Angew. Math. 474 (1996), 1-24. MR1390690 (98a:11063)

Department of Mathematics and Computer Science, University of Lethbridge, 4401 University Drive West, Lethbridge, Alberta, Canada T1K 3M4

E-mail address: akbary@cs.uleth.ca 